\title{
Autonomous Flight of the Rotorcraft-Based UAV Using RISE Feedback and NN Feedforward Terms
}

\author{
Jongho Shin, H. Jin Kim, Youdan Kim, and Warren E. Dixon
}

\begin{abstract}
A position tracking control system is developed for a rotorcraft-based unmanned aerial vehicle (RUAV) using robust integral of the signum of the error (RISE) feedback and neural network (NN) feedforward terms. While the typical NN-based adaptive controller guarantees uniformly ultimately bounded stability, the proposed NN-based adaptive control system guarantees semiglobal asymptotic tracking of the RUAV using the RISE feedback control. The developed control system consists of an inner-loop and outer-loop. The inner-loop control system determines the attitude of the RUAV based on an adaptive NN-based linear dynamic model inversion (LDI) method with the RISE feedback. The outer-loop control system generates the attitude reference corresponding to the given position, velocity, and heading references, and controls the altitude of the RUAV by the LDI method with the RISE feedback. The linear model for the LDI is obtained by a linearization of the nonlinear RUAV dynamics during hover flight. Asymptotic tracking of the attitude and altitude states is proven by a Lyapunov-based stability analysis, and a numerical simulation is performed on the nonlinear RUAV model to validate the effectiveness of the controller.
\end{abstract}

Index Terms-Adaptive position tracking control system, asymptotic stability, neural networks (NNs), robust integral of the signum of the error (RISE) feedback, rotorcraft-based unmanned aerial vehicle (RUAV).

$\begin{array}{ll}x^{S}, y^{S}, z^{S} & \begin{array}{l}\text { Position about the spatial coordinate } \\ \text { (measurable). }\end{array} \\ u, v, w & \begin{array}{l}\text { Velocity in } x, y \text { and } z \text {-direction about the body } \\ \text { coordinate (measurable). }\end{array} \\ p, q, r & \begin{array}{l}\text { Roll, pitch, and yaw rate about the body } \\ \text { coordinate (measurable). }\end{array} \\ \phi, \theta, \psi & \text { Roll, pitch, and yaw angles (measurable). } \\ a_{1}, b_{1} & \begin{array}{l}\text { Longitudinal and lateral flapping angles of } \\ \text { main rotor (unmeasurable). }\end{array} \\ g, m & \text { Gravity acceleration, mass of the RUAV. } \\ X_{\text {col }}, X_{\text {tail }} & \begin{array}{l}\text { Main- and tail-rotor collective sticks. } \\ X_{\text {lon }}, X_{\text {lat }}\end{array} \\ F_{x}, F_{y}, F_{z} & \begin{array}{l}\text { Longitudinal and lateral cyclic sticks. } \\ \text { Force in } x, y, \text { and } z \text {-direction about the body } \\ \text { coordinate. }\end{array}\end{array}$

Manuscript received March 14, 2011; accepted June 01, 2011. Manuscript received in final form June 15, 2011. Date of publication August 08, 2011; date of current version June 28, 2012. Recommended by Associate Editor C. A. Rabbath. This work was supported by the National Research Foundation of Korea (NRF) grant funded by the Korea government (MEST) (2010-0018871).

J. Shin, H. J. Kim, and Y. Kim are with the School of Mechanical and Aerospace Engineering and the Institute of Advanced Aerospace Technology, Seoul National University, Seoul 151-744, The Republic of Korea (e-mail: jh0524@snu.ac.kr; hjinkim@snu.ac.kr; ydkim@snu.ac.kr).

W. E. Dixon is with the Department of Mechanical and Aerospace Engineering, University of Florida, Gainesville, FL 32611-6250 USA (e-mail: wdixon@ufl.edu).

Color versions of one or more of the figures in this brief are available online at http://ieeexplore.ieee.org.

Digital Object Identifier 10.1109/TCST.2011.2160179

\section{INTRODUCTION}

\section{A} ROTORCRAFT-BASED unmanned aerial vehicle (RUAV) is a versatile machine which can perform hover and vertical take-off and landing (VTOL) maneuvers. These characteristics have led to interests in the deployment of autonomous RUAV for military and civilian applications. Such applications involve low-speed tracking maneuvers in law-enforcement, reconnaissance, and operations where no runway is available for take-off and landing. While a fixed-wing aircraft is generally internally stable [1], [2], the RUAV dynamics are naturally unstable [3]-[5] without closed-loop control, which makes the control system design for the RUAV more challenging. To effectively overcome this difficulty, various control methods have been studied such as robust control [6], proportional-integral-differential (PID) control [7], model predictive control [8], [9], adaptive control [10]-[12], and learning-based control [13].

The RUAV control system can usually be divided into two parts, similar to the fixed-wing UAV control system: an inner-loop for controlling the attitude of the RUAV and an outer-loop for generating the attitude reference corresponding to the given position and velocity references [1], [2]. An altitude tracking controller is also designed for the vertical axis. Therefore, the dynamic behavior of the RUAV is largely determined by the inner-loop control system. Performance improvements of the attitude control system have been enabled through adaptive control. In particular, neural network (NN)-based adaptive control methods have shown excellent performance in the presence of uncertainties through various simulations and flight tests [10], [11]. In addition, the altitude control system has been also designed by augmenting adaptive NN terms [12]. NN-based methods have proven to be useful, yet the function approximation yields uniformly ultimate bounded results because of the residual approximation error. A simple method for removing the inherent error is to augment the $\mathrm{NN}$ with a robustifying term such as sliding mode control, but this approach leads to a discrete control law [14], [15].

To improve the performance of the RUAV control system with a continuous control law, this study and the preliminary work in [16] propose the NN-based adaptive control system with robust integral of the signum of the error (RISE) feedback [17]-[22]. The RISE feedback control can compensate for disturbances or uncertainty while ensuring a semi-global asymptotic results with a continuous controller. When augmented with an adaptive feedforward term, the sufficient high gain conditions of the RISE feedback are reduced, and the performance of the combined methods has been validated through simulation and experiment [17]-[19]. While the adaptive terms in [17] and [19] have been designed using a general adaptive control concept [14], a 
NN-based adaptive feedforward term is used in [18]. In this research, a linear dynamic model inversion (LDI) method is employed to reduce the design load of the control gain for the RISE feedback and adaptive feedforward term. The advantage of the LDI method is that, by utilizing a nominal model information, the need for heavier design efforts and large control gains for the RISE feedback can be avoided.

The proposed RUAV control system is divided into the outer-loop and inner-loop control systems. The outer-loop control system is composed of translational dynamics inversion and altitude control. In the translational dynamics inversion terms, roll and pitch commands $\left(\phi_{\text {ref }}, \theta_{\text {ref }}\right)$ are generated corresponding to the given position $\left(x_{\text {ref }}^{S}, y_{\text {ref }}^{S}, z_{\text {ref }}^{S}\right)$, velocity $\left(\dot{x}_{\text {ref }}^{S}, \dot{y}_{\text {ref }}^{S}, \dot{z}_{\text {ref }}^{S}\right)$, and heading commands $\left(\psi_{\text {ref }}\right)$, [23], [24], where $(\cdot)_{\text {ref }}$ denotes the reference. To follow the given $z_{\text {ref }}^{S}$, $\phi_{\text {ref }}, \theta_{\text {ref }}$, and $\psi_{\text {ref }}$, altitude and inner-loop control systems are designed by the LDI method with the RISE feedback. The linear model for LDI is obtained by linearization of the nonlinear RUAV model at hover flight. The altitude and attitude tracking control systems are designed by differentiating $z^{S}, \phi, \theta$, and $\psi$ twice with respect to time. Therefore, their second time derivatives have to include the control inputs for designing the LDI. That is, the input gain matrix obtained in the linearization has to be invertible because the LDI method requires an inversion of the input gain matrix. However, while the control inputs $X_{\mathrm{col}}$ and $X_{\text {tail }}$ directly affect the dynamics on the vertical velocity $(w)$ and yaw rate $(r)$, the dynamics on the pitch rate $(q)$ and roll rate $(p)$ are determined only by the flapping angles $a_{1}, b_{1}$ and other states. The longitudinal and lateral cyclic control inputs $X_{\text {lon }}$ and $X_{\text {lat }}$ directly decide the motion of $a_{1}$ and $b_{1}$ only [3]-[5]. To invert the input gain matrix, this study rearranges it such that the dynamics of $q$ and $p$ are directly influenced by $X_{\text {lon }}$ and $X_{\text {lat }}$, and the dynamic effect on $a_{1}$ and $b_{1}$ is included in the uncertainty. Because this rearrangement increases the uncertainty that has to be removed by the RISE feedback, the NN-based adaptive feedforward term is added to the inner-loop control system to reduce the load of the RISE feedback. An adaptive rule for the NN approximator is designed to permit zero initial values by injecting more information (NN estimation terms) into the adaptive rule, without the need to design the initial values of the $\mathrm{NN}$ estimator [18].

This brief is organized as follows. In Section II, the nonlinear RUAV model is reviewed briefly and linear models for the attitude and altitude control systems are obtained at the hover condition. In Section III, we design the NN-based adaptive control system with RISE feedback and prove semi-global asymptotic tracking using a Lyapunov stability analysis. A detailed numerical simulation result is presented in Section IV. Finally, conclusions are included in Section V.

\section{DYNAMIC MODEL OF RUAV}

This section describes the general nonlinear dynamic model of the RUAV and its linearization at hover flight. Then, to design the attitude and altitude control systems separately, the linearized system is divided into two subsystems: the first one for the attitude control and the other for the altitude control system.
Generally, the dynamics for the RUAV are given as

$$
\dot{\mathbf{x}}(t)=f(\mathbf{x}(t), \mathbf{u}(t))
$$

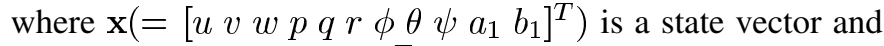
$\mathbf{u}\left(=\left[X_{\text {col }} X_{\text {lon }} X_{\text {lat }} X_{\text {tail }}\right]^{T}\right)$ is a control input vector [3]. The kinematics are represented as

$$
\begin{aligned}
\dot{\mathbf{x}}_{1} & =\Phi\left(\mathbf{x}_{1}\right) \mathbf{x}_{2} \\
{\left[\dot{x}^{S} \dot{y}^{S} \dot{z}^{S}\right]^{T} } & =L^{B \rightarrow S}[u v w]^{T}
\end{aligned}
$$

where $\mathbf{x}_{1}=[\phi \theta \psi]^{T}, \mathbf{x}_{2}=[p q r]^{T}$, and $\Phi\left(\mathbf{x}_{1}\right)$ and $L^{B \rightarrow S}$ are the direction cosine and transformation matrices from the body coordinate $B$ to the spatial coordinate $S$, respectively [1].

To derive our control law, the dynamic equations in (1) are linearized at hover flight condition

$$
\begin{aligned}
& \dot{\mathbf{x}}=A \mathbf{x}+B \mathbf{u}+d \\
& \mathbf{y}=C \mathbf{x}
\end{aligned}
$$

where $A$ and $B$ are the Jacobian matrices for a given flight condition [3], $\mathbf{y}=[u v w p q r \phi \theta \psi]^{T}$ is a measurable output, and $C$ is the output matrix for extracting $\mathbf{y}$ from the state vector $\mathrm{x}$. The term $d$ is considered as the uncertainty, which is composed of trim information and high order term derived by the linearization. It is defined as $d \triangleq-A \mathbf{x}_{0}-B \mathbf{u}_{0}+\Theta$, where the subscript zero denotes a trim value, and $\Theta$ is a high order term.

In this study, the attitude and altitude control systems are designed separately using (4) and (5). To facilitate this, the matrices $A, B$ in (4) are divided into two parts: one for the attitude control and one for the altitude control. This is motivated by the fact that the attitude states $\mathbf{x}_{1}$ of the RUAV are mainly connected to the control input $\mathbf{u}_{a}$ which is composed of $X_{\text {lon }}$, $X_{\text {lat }}$ and $X_{\text {tail }}$, while the altitude dynamics are dominated by the main-rotor collective stick $X_{\mathrm{col}}$ [5].

\section{A. Dynamic Equation for the Attitude Control System}

The attitude states $\mathbf{x}_{1}$ are mainly dominated by the control input $\mathbf{u}_{a}$ [5]. To utilize this property in the attitude control system design, the attitude states are partitioned as

$$
\mathbf{x}_{1} \triangleq C_{1} \mathbf{x}, \quad \mathbf{x}_{2} \triangleq C_{2} \mathbf{x}
$$

where $C_{1}, C_{2}$ are output matrices for extracting $\mathbf{x}_{1}$ and $\mathbf{x}_{2}$ from the state vector $\mathbf{x}$, respectively. Differentiating (2) yields

$$
\begin{aligned}
\ddot{\mathbf{x}}_{1}= & \dot{\Phi}\left(\mathbf{x}_{1}\right) \mathbf{x}_{2}+\Phi\left(\mathbf{x}_{1}\right) C_{2}\left(A \mathbf{x}+B_{a} \mathbf{u}_{a}+B_{w} X_{\mathrm{col}}+d\right) \\
= & \dot{\Phi}\left(\mathbf{x}_{1}\right) \mathbf{x}_{2}+\bar{A}_{a} \mathbf{y}+\Phi\left(\mathbf{x}_{1}\right) C_{2} B_{w} X_{\mathrm{col}}+\bar{B}_{a}\left(\mathbf{u}_{a}-\mathbf{u}_{a_{0}}\right) \\
& +\Delta_{a}
\end{aligned}
$$

where $\mathbf{u}_{a} \triangleq\left[X_{\text {lon }}, X_{\text {lat }}, X_{\text {tail }}\right]^{T}, \bar{A}_{a} \triangleq \Phi\left(\mathbf{x}_{1}\right) C_{2} A C^{\dagger}, \bar{B}_{a} \triangleq$ $\Phi\left(\mathbf{x}_{1}\right) C_{2} B_{a}, \Delta_{a} \triangleq \Phi\left(\mathbf{x}_{1}\right) C_{2}\left(d+B_{a} \mathbf{u}_{a_{0}}\right)$, the subscript $a$ denotes attitude, $\mathbf{u}_{a_{0}}$ is trim control input at hover condition, $B_{a}$ is a submatrix of the matrix $B$ corresponding to the control input $\mathbf{u}_{a}, B_{w}$ is a submatrix of the matrix $B$ corresponding to the control input $X_{\text {col }}$, and $C^{\dagger}\left(\in \Re^{11 \times 9}\right)$ is the pseudo inverse of $C$ which is composed of the identity matrix $\left(\in \Re^{9 \times 9}\right)$ and zero matrix $\left(\in \Re^{2 \times 9}\right)$. The term $\dot{\Phi}\left(\mathbf{x}_{1}\right)$ is computed using (2) and 
$X_{\mathrm{col}}$ is designed in the altitude control system. Therefore, all the elements in (7) are known except for the uncertainty $\Delta_{a}$.

The remaining concern is that $\bar{B}_{a}$ is a matrix of rank 1 because while the control input $X_{\text {tail }}$ directly affects the dynamics on the yaw rate $(r)$, the dynamics on the pitch rate $(q)$ and roll rate $(p)$ are not directly connected to the longitudinal and lateral cyclic sticks $X_{\text {lon }}$ and $X_{\text {lat }}$. As shown in [3]-[5], $X_{\text {lon }}$ and $X_{\text {lat }}$ determine just the motion of the flapping angles $a_{1}, b_{1}$, and the dynamics on the pitch rate $(q)$ and roll rate $(p)$ are determined by $a_{1}, b_{1}$ and other states only. Since the inverse of $\bar{B}_{a}$ is required for the LDI, $\bar{B}_{a}$ is rearranged such that the control effectiveness for $a_{1}$ and $b_{1}$ dynamics with respect to $X_{\text {lon }}, X_{\text {lat }}$ directly affects $q$ and $p$. It is achieved by substituting the steady state flapping angles into $q$ and $p$ dynamics, which is obtained by setting the time derivative $\dot{a}_{1}, \dot{b}_{1}$ to zero in the flapping dynamics [5]. Then, it is possible to invert the matrix $\bar{B}_{a}$.

\section{B. Dynamic Equation for the Altitude Control System}

While the longitudinal and lateral cyclic sticks affect just the motion of the flapping angles $a_{1}$ and $b_{1}$, the dynamics of the vertical velocity $w$ is directly affected by the main rotor collective stick input $X_{\text {col }}$ [3]. Thus, the LDI method can be directly applied to the design of the altitude control system, unlike the attitude control system.

To design the LDI-based altitude controller, the following differential equation is developed using (3) [1]:

$$
\dot{z}^{S}=-u \sin \theta+v \sin \phi \cos \theta+w \cos \phi \cos \theta .
$$

The time derivative of (8) can be written as

$$
\ddot{z}^{S}=f_{w}+\dot{w} \cos \phi \cos \theta+\Delta
$$

where $\Delta \triangleq-\dot{u} \sin \theta+\dot{v} \sin \phi \cos \theta, f_{w} \triangleq-u \dot{\theta} \cos \theta$ $+v \dot{\phi} \cos \phi \cos \theta-v \dot{\theta} \sin \phi \sin \theta-w \dot{\phi} \sin \phi \cos \theta-w \dot{\theta} \cos \phi \sin \theta$.

Then, (9) is rearranged as

$$
\begin{aligned}
\ddot{z}^{S}= & f_{w}+C_{w}\left(A \mathbf{x}+B_{w} X_{\mathrm{col}}+B_{a} \mathbf{u}_{a}+d\right) \times \cos \phi \cos \theta \\
& +\Delta \\
= & f_{w}+\left(\bar{A}_{w} \mathbf{y}+\bar{B}_{w}\left(X_{\mathrm{col}}-X_{\mathrm{col}_{0}}\right)\right) \times \cos \phi \cos \theta \\
& +\Delta_{w}
\end{aligned}
$$

where $\bar{A}_{w} \triangleq C_{w} A C^{\dagger}, \bar{B}_{w} \triangleq C_{w} B_{w}, \Delta_{w} \triangleq C_{w}(d+$ $\left.B_{w} X_{\operatorname{col}_{0}}\right) \cos \phi \cos \theta+\Delta, C_{w}$ is a output matrix for extracting $w$ from $\mathbf{x}$, and $X_{\mathrm{col}_{0}}$ is trim control input at hover condition. Since $C_{w} B_{a}$ becomes a zero row vector [5], the effect with the control input $\mathbf{u}_{a}$ is removed. As pointed out previously, the control effectiveness $\bar{B}_{w}$ of $X_{\mathrm{col}}$ is a real constant value because $X_{\mathrm{col}}$ directly affects the dynamics on the vertical velocity $w$. Thus, the LDI method can be directly utilized for the altitude control system with $\bar{B}_{w}$. To design the attitude and altitude control systems with the RISE feedback, we need the following assumption on the uncertainties $\Delta_{a}, \Delta_{w}$ in (7) and (10).

Assumption 1: The nonlinear uncertainties $\Delta_{a}, \Delta_{w}$ are the functions of $\mathbf{x}_{1}, \dot{\mathbf{x}}_{1}$ and $z^{S}, \dot{z}^{S}$, respectively, i.e., $\Delta_{a} \triangleq \Delta_{a}\left(\mathbf{x}_{1}, \dot{\mathbf{x}}_{1}\right), \Delta_{w} \triangleq \Delta_{w}\left(z^{S}, \dot{z}^{S}\right)$. Then, if $\mathbf{x}_{1}, \dot{\mathbf{x}}_{1}, z^{S}$ and $\dot{z}^{S} \in \mathcal{L}_{\infty}, \Delta_{a}$ and $\Delta_{w}$ are bounded, and the first and second partial derivatives of $\Delta_{a}$ (with respect to $\mathbf{x}_{1}$ and $\dot{\mathbf{x}}_{1}$ ) and $\Delta_{w}$ (with respect to $z^{S}$ and $\dot{z}^{S}$ ) exist and are bounded.

In (7) and (10), $\Delta_{a}$ is composed of $\mathbf{x}_{1}$, constant trim states and high order terms, while $\Delta_{w}$ is mainly dominated by constant trim states and high order terms under small pitch and roll angles. This means that they are deviation from the specific trim point, and their magnitude is very small comparing to the known terms in (7) and (10). Therefore, $\Delta_{a}$ and $\Delta_{w}$ are dominated by Euler angle, their rates and vertical position, its velocity, respectively, because they are just small uncertainties generated in the motion of the rotational and vertical acceleration. Moreover, the fact that any planar translational motion of the RUAV is obtained through rolling or pitching the aircraft [5], and the control system of the RUAV can be properly designed by decoupling the vertical and translational motions [6], [7] makes the above assumption reasonable.

\section{LDI-BASED NN ADAPTIVE CONTROL FOR THE RUAV WITH RISE FEEDBACK}

This section presents the LDI-based NN adaptive control for the RUAV with the RISE feedback. The objective of the proposed control system is to follow the given position $\left(x_{\text {ref }}^{S}, y_{\text {ref }}^{S}, z_{\text {ref }}^{S}\right)$, velocity $\left(\dot{x}_{\text {ref }}^{S}, \dot{y}_{\text {ref }}^{S}, \dot{z}_{\text {ref }}^{S}\right)$ and heading commands $\left(\psi_{\text {ref }}\right)$. The control system is divided into outer-loop and inner-loop control systems. In the outer-loop control system, the reference attitudes $\phi_{\text {ref }}$ and $\theta_{\text {ref }}$ are generated corresponding to the given position, velocity, and heading commands [23], [24], and the altitude controller is designed using the LDI-based RISE feedback control. Then, the LDI-based NN adaptive control with the RISE feedback for the inner-loop system is designed to track $\phi_{\text {ref }}, \theta_{\text {ref }}$, and $\psi_{\text {ref }}$.

\section{A. Design of the Outer-Loop Control System}

The outer-loop control system is divided into two parts: the translational dynamics inversion and altitude control. The translational dynamics inversion component generates $\phi_{\text {ref }}$ and $\theta_{\text {ref }}$, while the altitude control system is designed by the LDI method with the RISE feedback.

1) Translational Dynamics Inversion for $\phi_{\mathrm{ref}}$ and $\theta_{\mathrm{ref}}$ : Generally, the relationship between the accelerations $\left(\ddot{x}^{S}, \ddot{y}^{S}, \ddot{z}^{S}\right)$ in the spatial coordinate frame and accelerations $\left(F_{x} / m, F_{y} / m, F_{z} / m\right)$ in the body coordinate frame is expressed using the transformation matrix $L^{B \rightarrow S}$ in (3) [1]. If $\ddot{x}^{S}, \ddot{y}^{S}$ and $\ddot{z}^{S}$ are substituted for the pseudo control variables $v_{x}, v_{y}$, and $v_{z}$, respectively, the following equation holds:

$$
\left[\begin{array}{lll}
v_{x} & v_{y} & v_{z}
\end{array}\right]^{T}+\left[\begin{array}{lll}
0 & 0 & -g
\end{array}\right]^{T}=L^{B \rightarrow S}\left[\frac{F_{x}}{m} \frac{F_{y}}{m} \frac{F_{z}}{m}\right]^{T} .
$$

Since the forces generated by the cyclic sticks $X_{\text {lon }}, X_{\text {lat }}$ and tail rotor collective stick $X_{\text {tail }}$ are relatively smaller than the force due to the main rotor collective stick $X_{\text {col }}$, the forces $F_{x}$, $F_{y}, F_{z}$ are approximated as $\tilde{F}_{x}, \tilde{F}_{y}, \tilde{F}_{z}$ which are related to the main rotor collective stick $X_{\text {col }}$ only. Rearranging (11) yields

$$
\begin{gathered}
\left(\frac{\tilde{F}_{x}}{m}\right)^{2}+\left(\frac{\tilde{F}_{y}}{m}\right)^{2}+\left(\frac{\tilde{F}_{z}}{m}\right)^{2} \\
=v_{x}^{2}+v_{y}^{2}+\left(v_{z}-g\right)^{2}
\end{gathered}
$$




$$
\begin{aligned}
& \frac{\tilde{F}_{y}}{m} \cos \phi_{\text {ref }}-\frac{\tilde{F}_{z}}{m} \sin \phi_{\text {ref }} \\
& =-v_{x} \sin \psi_{\text {ref }}+v_{y} \cos \psi_{\text {ref }} \\
& \frac{\tilde{F}_{x}}{m}=\left(v_{x} \cos \psi_{\text {ref }}+v_{y} \sin \psi_{\text {ref }}\right) \cos \theta_{\text {ref }} \\
& \quad-\left(v_{z}-g\right) \sin \theta_{\text {ref }}
\end{aligned}
$$

where the reference signal $\psi_{\text {ref }}$ is given. To obtain an approximate value of $\phi_{\text {ref }}$ and $\theta_{\text {ref }}$ analytically, $\tilde{F}_{x}$ and $\tilde{F}_{y}$ can be ignored because they are relatively smaller than $\tilde{F}_{z}$. Based on this assumption [23], [24]

$$
\begin{aligned}
\left(\frac{\tilde{F}_{z}}{m}\right)^{2} & \triangleq v_{x}^{2}+v_{y}^{2}+\left(v_{z}-g\right)^{2} \\
\phi_{\text {ref }} & \triangleq \arcsin \left(\frac{v_{x} \sin \psi_{\text {ref }}-v_{y} \cos \psi_{\text {ref }}}{\frac{\tilde{F}_{z}}{m}}\right) \\
\theta_{\text {ref }} & \triangleq \arctan \left(\frac{v_{x} \cos \psi_{\text {ref }}+v_{y} \sin \psi_{\text {ref }}}{v_{z}-g}\right) .
\end{aligned}
$$

To define the pseudo control variables $v_{x}, v_{y}, v_{z}$, the error in each axis of the spatial coordinate is defined as

$$
e_{x} \triangleq x_{\mathrm{ref}}^{S}-x^{S}, e_{y} \triangleq y_{\mathrm{ref}}^{S}-y^{S}, e_{z} \triangleq z_{\mathrm{ref}}^{S}-z^{S} .
$$

Then, PD-type pseudo control variables are defined as

$$
\begin{aligned}
& v_{x} \triangleq k_{x}^{P} e_{x}+k_{x}^{D} \dot{e}_{x} \\
& v_{y} \triangleq k_{y}^{P} e_{y}+k_{y}^{D} \dot{e}_{y} \\
& v_{z} \triangleq k_{z}^{P} e_{z}+k_{z}^{D} \dot{e}_{z}
\end{aligned}
$$

where the proportional $\left(k_{i}^{P}\right)$ and derivative $\left(k_{i}^{D}\right)$ gains are designed by satisfying the system requirement such as settling time, overshoot, etc., [15]. $\phi_{\text {ref }}, \theta_{\text {ref }}$ in (16) and the given $\psi_{\text {ref }}$ are used as the reference commands for the attitude control system.

To develop the altitude and attitude control systems, the first four time derivatives of $z_{\text {ref }}^{S}, \phi_{\text {ref }}, \theta_{\text {ref }}$, and $\psi_{\text {ref }}$ have to be bounded all the time. Therefore, we utilize the following assumption.

Assumption 2: To make $\phi_{\text {ref }}, \theta_{\text {ref }}$ and their time derivatives bounded all time, $u_{x}, u_{y}, u_{z}$, and $\psi_{\text {ref }}$ are designed to satisfy the following conditions:

$$
u_{z} \neq g, 2 u_{x} u_{y}\left(1-\frac{1}{2} \sin 2 \psi_{\text {ref }}\right) \leq\left(u_{z}-g\right)^{2} .
$$

The condition $u_{z} \neq g$ is reasonable because $u_{z}$ cannot be the same as $g$ unless the RUAV falls free. Then, the bounded time derivatives (up to the fourth-order) of $z_{\text {ref }}^{S}, \phi_{\text {ref }}, \theta_{\text {ref }}$, and $\psi_{\text {ref }}$ can be generated by fourth-order command filters.

2) LDI-Based Altitude Control Design With RISE Feedback: To design the altitude control system, the filtered error $r_{z}$ is defined as $r_{z} \triangleq \dot{v}_{z}+k_{z} v_{z}$, where $k_{z}$ is a positive constant. Using the dynamic (10) and $e_{z}$ and $v_{z}$ in (17) and (18), the following open-loop error equation can be determined:

$r_{z}=k_{z} v_{z}+k_{z}^{P} \dot{e}_{z}+k_{z}^{D} \ddot{z}_{\text {ref }}^{S}$

$$
\begin{aligned}
& -k_{z}^{D}\left(\bar{A}_{w} \mathbf{y}+\bar{B}_{w}\left(X_{\mathrm{col}}-X_{\mathrm{col}_{0}}\right)\right) \cos \phi \cos \theta \\
& -k_{z}^{D} f_{w}-k_{z}^{D} \Delta_{w} \\
= & S_{z}-k_{z}^{D}\left(\bar{A}_{w} \mathbf{y}+\bar{B}_{w}\left(X_{\mathrm{col}}-X_{\mathrm{col}_{0}}\right)\right) \cos \phi \cos \theta-k_{z}^{D} f_{w}
\end{aligned}
$$

where

$$
S_{z} \triangleq k_{z} v_{z}+k_{z}^{P} \dot{e}_{z}+k_{z}^{D} \ddot{z}_{\text {ref }}^{S}-k_{z}^{D} \Delta_{w} .
$$

The LDI-based altitude control law $X_{\text {col }}$ with the RISE feedback is defined as

$$
X_{\mathrm{col}} \triangleq \frac{1}{\bar{B}_{w}}\left(-\bar{A}_{w} \mathbf{y}+\frac{\mu_{z}-k_{z}^{D} f_{w}}{k_{z}^{D} \cos \phi \cos \theta}\right)+X_{\mathrm{col}_{0}}
$$

where $\bar{A}_{w} \mathbf{y} / \bar{B}_{w}$ and $k_{z}^{D} f_{w} / \bar{B}_{w}$ are used for eliminating the nominal model information, and $X_{\mathrm{col}_{0}}$ is the trim control input. The RISE feedback term $\mu_{z}$ is given as [20]

$$
\mu_{z}(t) \triangleq\left(k_{z}^{S}+1\right) v_{z}(t)-\left(k_{z}^{S}+1\right) v_{z}(0)+\eta_{z}
$$

where $\eta_{z}$ is the generalized solution to $\dot{\eta}_{z} \triangleq\left(k_{z}^{S}+1\right) k_{z} v_{z}(t)+$ $\beta_{z} \operatorname{sgn}\left(v_{z}\right)$, and $k_{z}^{S}, \beta_{z}$ are positive control gains.

\section{B. Design of the Inner-Loop Control System: LDI-Based NN Adaptive Control With the RISE Feedback}

To quantify the inner-loop control performance, a tracking error $\mathbf{e}_{a_{1}}(t)\left(\in \Re^{3}\right)$ is defined as $\mathbf{e}_{a_{1}} \triangleq \mathbf{x}_{a}^{d}-\mathbf{x}_{1}$, where $\mathbf{x}_{a}^{d}(=$ $\left.\left[\begin{array}{lll}\phi_{\text {ref }} & \theta_{\text {ref }} & \psi_{\text {ref }}\end{array}\right]^{T}\right)$ is the reference command vector. Filtered tracking errors $\mathbf{e}_{a_{2}}(t)$ and $\mathbf{r}_{a}(t)$ are also defined as

$$
\mathbf{e}_{a_{2}} \triangleq \dot{\mathbf{e}}_{a_{1}}+K_{1} \mathbf{e}_{a_{1}} \quad \mathbf{r}_{a} \triangleq \dot{\mathbf{e}}_{a_{2}}+K_{2} \mathbf{e}_{a_{2}}
$$

where $K_{1}, K_{2}$ are diagonal positive constant matrices.

Similar to the altitude control system, an open-loop error system is developed as

$$
\begin{aligned}
\mathbf{r}_{a}=K_{1} \dot{\mathbf{e}}_{a_{1}}+K_{2} \mathbf{e}_{a_{2}}+\ddot{\mathbf{x}}_{a}^{d} \\
-\left(\dot{\Phi}\left(\mathbf{x}_{1}\right) \mathbf{x}_{2}+\bar{A}_{a} \mathbf{y}+\bar{B}_{a}\left(\mathbf{u}_{a}-\mathbf{u}_{a_{0}}\right)\right)-\Delta_{a} .
\end{aligned}
$$

As explained in Section II-A, $\Delta_{a}$ includes not only the uncertainty related to measurable states but also the flapping angle dynamics. To reduce the load on the RISE feedback for eliminating the larger $\Delta_{a}$, the attitude control system includes the $\mathrm{NN}$ feedforward compensation. In a similar manner as in [18], the open-loop error system (25) is rearranged by adding an auxiliary function $\Delta_{a}^{d}\left(\mathbf{x}_{a}^{d}, \dot{\mathbf{x}}_{a}^{d}, \ddot{\mathbf{x}}_{a}^{d}\right)$

$$
\mathbf{r}_{a}=S_{a}-\left(\dot{\Phi}\left(\mathbf{x}_{1}\right) \mathbf{x}_{2}+\bar{A}_{a} \mathbf{y}+\bar{B}_{a}\left(\mathbf{u}_{a}-\mathbf{u}_{a_{0}}\right)\right)-\Delta_{a}^{d}
$$

where $\Delta_{a}^{d}(\cdot)$ is $\mathcal{C}^{2}$, and $S_{a}$ is defined as

$$
S_{a} \triangleq K_{1} \dot{\mathbf{e}}_{a_{1}}+K_{2} \mathbf{e}_{a_{2}}+\ddot{\mathbf{x}}_{a}^{d}-\Delta_{a}+\Delta_{a}^{d} .
$$

The auxiliary function $\Delta_{a}^{d}$ can be represented by a three-layer $\mathrm{NN}$ as

$$
\Delta_{a}^{d}=W^{T} \sigma\left(V^{T} \mathbf{x}_{d}\right)+\epsilon\left(\mathbf{x}_{d}\right)
$$

where $W\left(\in \Re^{N \times 3}\right)$ and $V\left(\in \Re^{10 \times N}\right)$ are bounded constant ideal weight matrices for representing $\Delta_{a}^{d}, N$ is the number of 


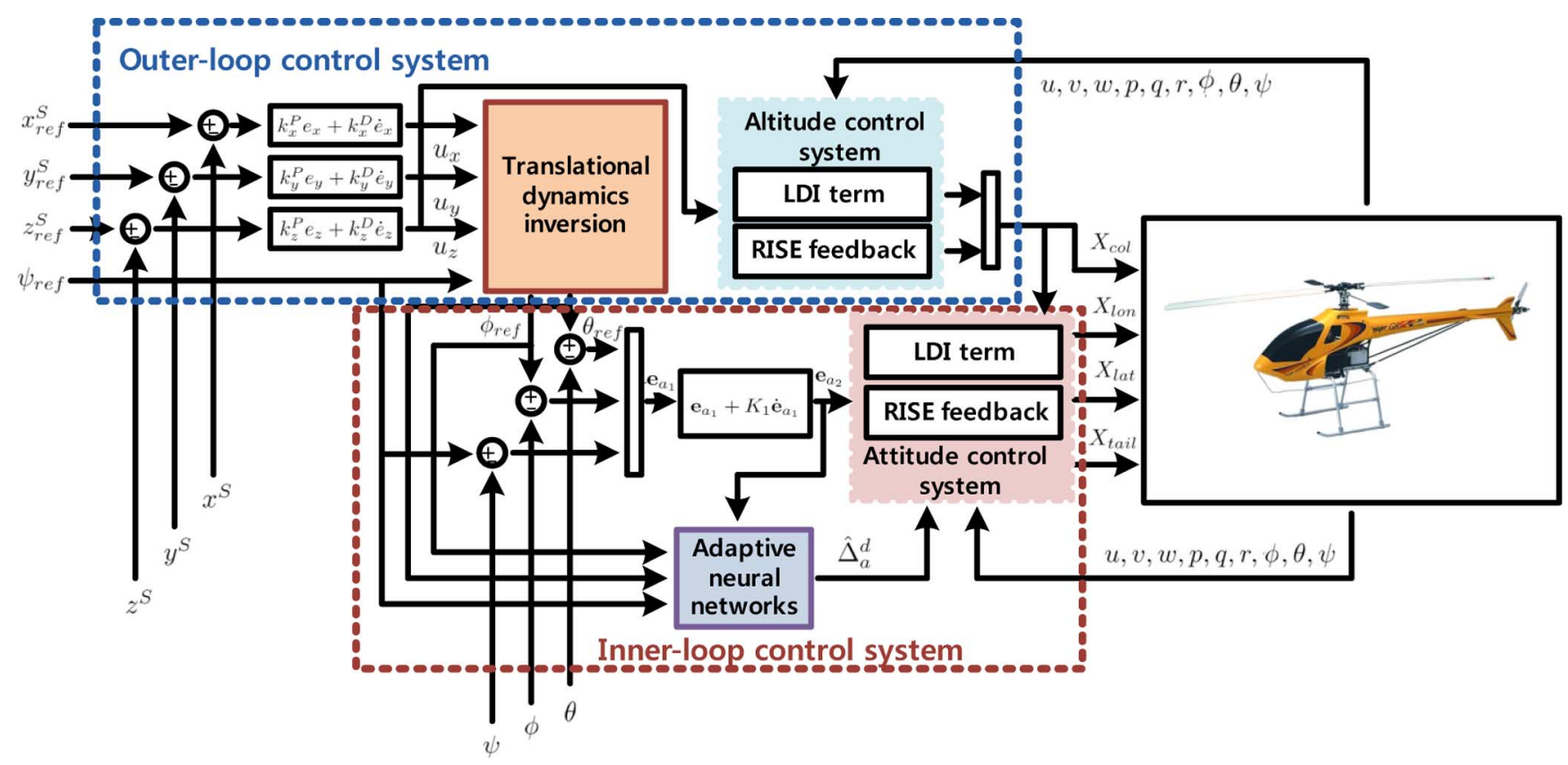

Fig. 1. Overall structure of the LDI-based adaptive control system for the RUAV with the RISE feedback.

the neurons in the hidden layer, $\sigma(\cdot)$ is the sigmoid activation function [18], $\epsilon\left(\mathbf{x}_{d}\right)$ is the inherent error of the $\mathrm{NN}$ estimator, and $\mathbf{x}_{d}\left(\in \Re^{10}\right)$ is defined as $\mathbf{x}_{d}(t) \triangleq\left[1, \mathbf{x}_{a}^{d}, \dot{\mathbf{x}}_{a}^{d}, \ddot{\mathbf{x}}_{a}^{d}\right]^{T}$. Since the desired command $\mathbf{x}_{d}$ is bounded in Assumption 2, $\epsilon\left(\mathbf{x}_{d}\right)$, $\dot{\epsilon}\left(\mathbf{x}_{d}, \dot{\mathbf{x}}_{d}\right)$ and $\ddot{\epsilon}\left(\mathbf{x}_{d}, \dot{\mathbf{x}}_{d}, \ddot{\mathbf{x}}_{d}\right)$ are bounded with real positive constants $\zeta_{\epsilon}, \zeta_{\dot{\epsilon}}$ and $\zeta_{\ddot{\epsilon}}$, respectively.

The LDI-based NN adaptive attitude control law with the RISE feedback $\mathbf{u}_{a}$ is defined as

$$
\begin{aligned}
\mathbf{u}_{a} \triangleq & \bar{B}_{a}^{-1}\left(-\bar{A}_{a} \mathbf{y}-\dot{\Phi}\left(\mathbf{x}_{1}\right) \mathbf{x}_{2}-\Phi\left(\mathbf{x}_{1}\right) C_{2} B_{w} X_{\mathrm{col}}-\hat{\Delta}_{a}^{d}+\mu_{a}\right) \\
& +\mathbf{u}_{a_{0}}
\end{aligned}
$$

where $\bar{B}_{a}^{-1}\left(\bar{A}_{a} \mathbf{y}+\dot{\Phi}\left(\mathbf{x}_{1}\right) \mathbf{x}_{2}\right)$ is used for eliminating the nominal model information, $\Phi\left(\mathbf{x}_{1}\right) C_{2} B_{w} X_{\mathrm{col}}$ cancels the effect of the main-rotor collective stick in the yaw rate dynamics [5], and $\mathbf{u}_{a_{0}}$ is the trim control input. The RISE feedback term $\mu_{a}\left(\in \Re^{3}\right)$ is defined as [20]

$$
\mu_{a}(t) \triangleq\left(K_{a}^{S}+\mathbb{\pi}_{3}\right) \mathbf{e}_{a_{2}}(t)-\left(K_{a}^{S}+\mathbb{1}_{3}\right) \mathbf{e}_{a_{2}}(0)+\eta_{a}
$$

where $\eta_{a}$ is the generalized solution to $\dot{\eta}_{a} \triangleq\left(K_{a}^{S}+\right.$ $\left.\mathbb{1}_{3}\right) K_{2} \mathbf{e}_{a_{2}}(t)+\beta_{a_{1}} \operatorname{sgn}\left(\mathbf{e}_{a_{2}}\right)$, the diagonal matrices $K_{a}^{S}, \beta_{a_{1}}$ are chosen by the stability analysis, and $\mathbb{I}_{3}$ is a $3 \times 3$ identity matrix. The NN feedforward term $\hat{\Delta}_{a}^{d}$ in (29) is generated as

$$
\hat{\Delta}_{a}^{d} \triangleq \hat{W}^{T} \sigma\left(\hat{V}^{T} \mathbf{x}_{d}\right)
$$

where the adaptation rules for the weight matrices $\hat{W}$ and $\hat{V}$ are given by

$$
\begin{aligned}
& \dot{\hat{W}} \triangleq \operatorname{Proj}\left(\hat{W}, \Gamma_{1}\left(\hat{\sigma}-\hat{\sigma}^{\prime} \hat{V} \dot{\mathbf{x}}_{d}\right) \mathbf{e}_{a_{2}}^{T}\right) \\
& \dot{\hat{V}} \triangleq \operatorname{Proj}\left(\hat{V}, \Gamma_{2} \dot{\mathbf{x}}_{d}\left(\hat{\sigma}^{\prime T} \hat{W} K_{2} \mathbf{e}_{a_{2}}\right)^{T}\right)
\end{aligned}
$$

$\hat{\sigma}=\sigma\left(\hat{V}^{T} \mathbf{x}_{d}\right), \hat{\sigma}^{\prime}=d \sigma(z) /\left.d z\right|_{z=\hat{V}^{T} \mathbf{x}_{d}}, \Gamma_{1}$, and $\Gamma_{2}$ are constant positive definite symmetric matrices, and $\operatorname{Proj}(\cdot, \cdot)$ is a projection operator [14]. The overall control system proposed in this study is given in Fig. 1 and its stability is briefly analyzed in the Appendix.

The adaptation rules (32) include $\hat{\sigma}$, which enables the zero initial value of the weight matrices $\hat{W}$ and $\hat{V}$, unlike the adaptive rule proposed in [18] where the estimation terms always become zeros if the initial weight values are set to zeros. Since the design of the initial matrix $\hat{W}$ or $\hat{V}$ is not trivial, the fact that the initial values of $\hat{W}$ and $\hat{V}$ can be chosen as zero matrices is a desirable property. In addition, the term $\hat{\sigma}$ improves the transient characteristics of the adaptation process because more information is utilized in the adaptation rule, as illustrated in Section IV.

\section{Simulation Results}

In this section, numerical simulation is performed using the nonlinear RUAV model to validate the performance of the proposed control system. The nonlinear RUAV model has been obtained from the flight test data [25].

To design the LDI-based control law, the nonlinear RUAV model is linearized at the following trim condition for hover flight:

states trim: $u, v, w, p, q, r, \theta, \psi=0, \phi=3.99 \mathrm{deg}, h=40 \mathrm{~m}$

input trim: $X_{\mathrm{col}}=0.085, X_{\text {lon }}=0.000, X_{\text {lat }}=0.006$

$$
X_{\text {tail }}=-0.199
$$

where the nonzero trim value of $\phi$ is for eliminating the antitorque generated by the main-rotor and, thus, $X_{\text {lat }}$ and $X_{\text {tail }}$ have nonzero trim input values.

The objective of the numerical simulation is to perform an automatic landing maneuver, where the scenario is given in Fig. 2. At the first step, the heading of the RUAV is controlled from 0 to $45 \mathrm{deg}$, which is determined by the initial position $(0,0,40)$ and reference position $(20,20,40)$. In the second step, the RUAV 


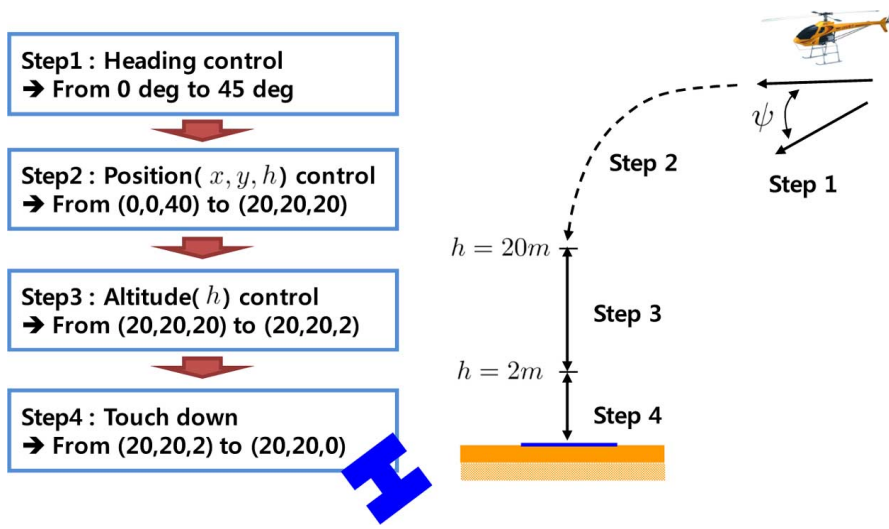

Fig. 2. Automatic landing scenario.

flies from $(0,0,40)$ to $(20,20,20)$. Then, the RUAV vertically descends to $2 \mathrm{~m}$ and touches down.

The design parameters for the proposed control system have to satisfy the condition that minimal values for $k_{z}^{P}, K_{1}, k_{z}$, and $K_{2}$ are set larger than $1 / 2,1 / 2,1$ and $1+\beta_{\alpha_{2}}$, respectively, and $k_{z}^{S}$ and $K_{a}^{S}$ are sufficiently large, as addressed in the Appendix and [18]. With these conditions, the control design parameters are chosen as

$$
\begin{aligned}
k_{x}^{P} & =k_{y}^{P}=0.188 \\
k_{x}^{D} & =k_{y}^{D}=0.613 \\
k_{z}^{P} & =0.6 \\
k_{z}^{D} & =0.6 \\
k_{z} & =1.1 \\
k_{z}^{S} & =2 \\
\beta_{z} & =0.01 \\
K_{1} & =\operatorname{diag}([4,5,0.6]) \\
K_{2} & =\operatorname{diag}([4,5,1.1]) \\
K_{a}^{S} & =\operatorname{diag}([3,3,5]) \\
\beta_{a_{1}} & =\operatorname{diag}([0.01,0.01,0.01])
\end{aligned}
$$

where the proportional and derivative gains for the pseudo control variables $v_{x}, v_{y}$ are designed for a settling time of $15 \mathrm{~s}$ and the maximum overshoot of $4 \%$. The number of the neurons in the hidden layer, $N$, is set to 5 and the initial values of $\hat{W}$ and $\hat{V}$ are set to zero matrices.

The results of the position tracking are given in Figs. 3 and 4. The tracking performance of the LDI-based NN adaptive control with the RISE feedback is satisfactory, and the attitude $\phi$ and $\theta$ of the RUAV follows the reference commands generated in the outer-loop control system as shown in Fig. 5. Although there is a small deviation of the yaw angle $\psi$ caused by the descent of the RUAV, because the anti-torque by the main rotor is counteracted by the tail rotor collective stick, it is negligible. Fig. 6 shows the control input history.

The LDI-based RISE feedback control system with the above control gains, without the adaptation logic in the inner-loop control system, has failed the automatic landing maneuver of the RUAV. This is because the uncertainty $\Delta_{a}$, which includes the flapping angle dynamics, is too large to be compensated by

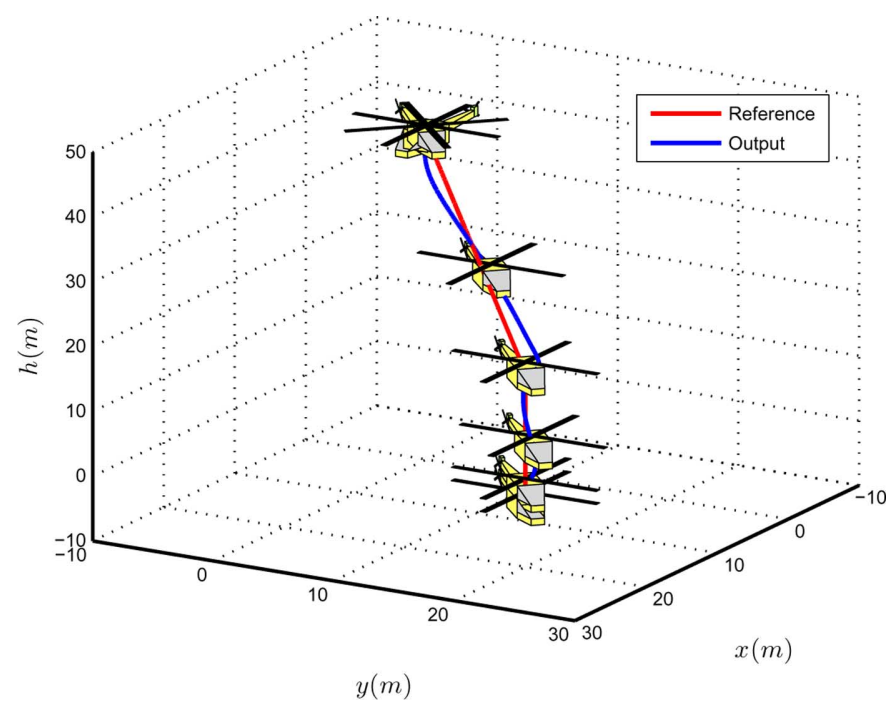

Fig. 3. Results of the position tracking in 3-D space.
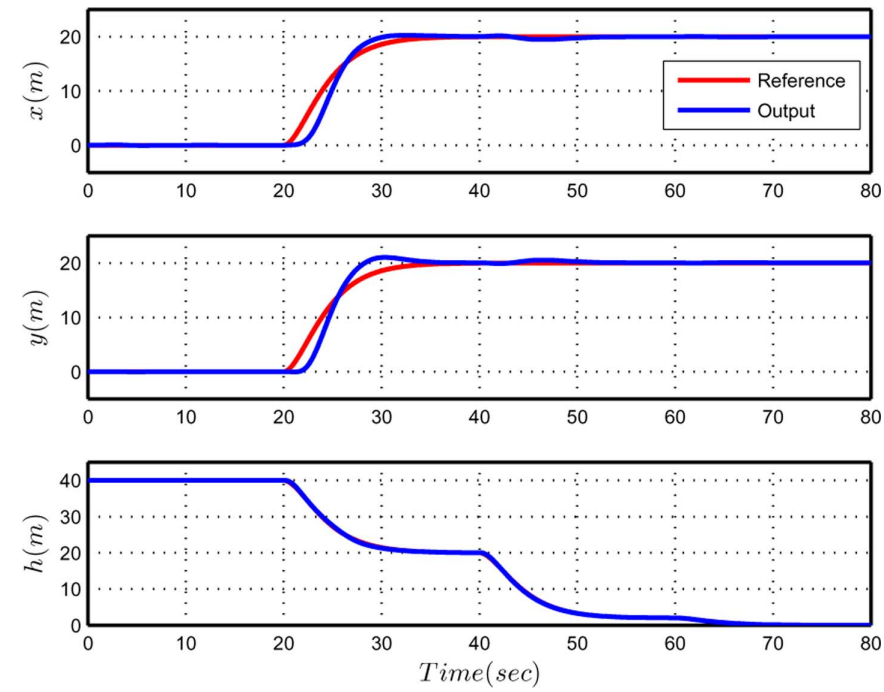

Fig. 4. Results of the position tracking.

the RISE feedback with (34) only. The adaptive rule without $\hat{\sigma}$ in [18], which needs the random selection of the initial weight values, has also made the RUAV unstable in the automatic landing maneuver, even though it could track the sinusodial attitude commands [16]. The reason is that, unlike the sinusodial reference presented in [16], the desired commands $\phi_{\text {ref }}$, $\theta_{\text {ref }}$ through the outer-loop control system are more difficult to follow using the NN estimation without $\hat{\sigma}$, based on the random initial value. Through the automatic landing simulation the performance of the LDI-based NN adaptive control with the RISE feedback has been validated, whose adaptation rules include $\hat{\sigma}$ and, thus, do not require efforts for choosing the initial values of the $\mathrm{NN}$ weight values.

By increasing the control gains of the RISE feedback, the automatic landing maneuver of the RUAV could be achieved without the NN feedforward terms. However, in this case, the RISE method yields high-gain sufficient conditions, thus, its performance can be significantly degraded in flight test, because there are sensor noise and sample time delays. As shown 
TABLE I

Root Mean Squared Error of the Position $(m)$ and Heading (Deg) Tracking Performance

\begin{tabular}{|c||c|c|c|c|}
\hline & $x_{r e f}-x$ & $y_{r e f}-y$ & $z_{r e f}-z$ & $\psi_{r e f}-\psi$ \\
\hline Only RISE feedback & 1.1319 & 1.1228 & 0.5040 & 4.4758 \\
\hline RISE feedback with NN & 0.9761 & 1.0473 & 0.5039 & 1.2912 \\
\hline
\end{tabular}
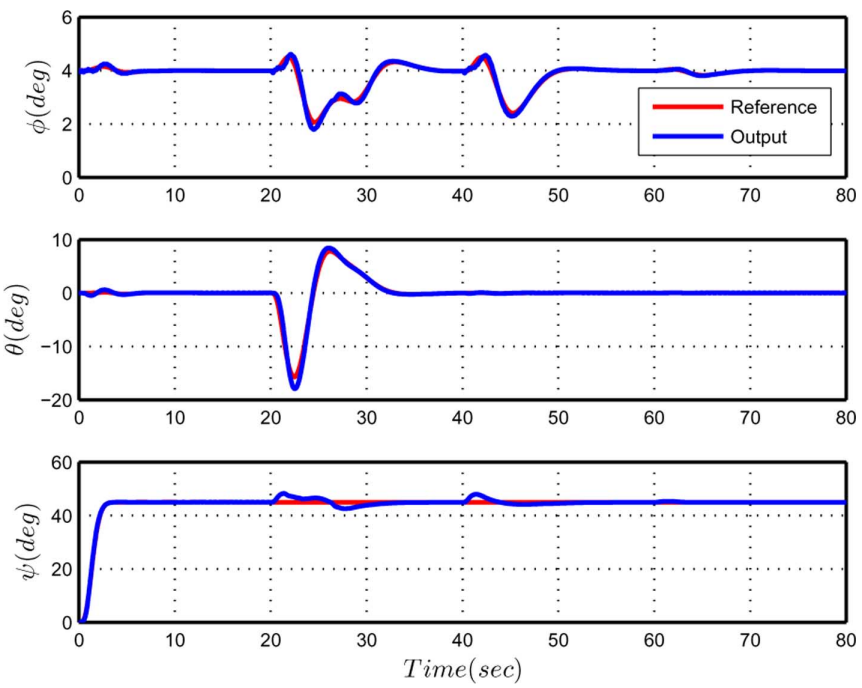

Fig. 5. Results of the euler angles.
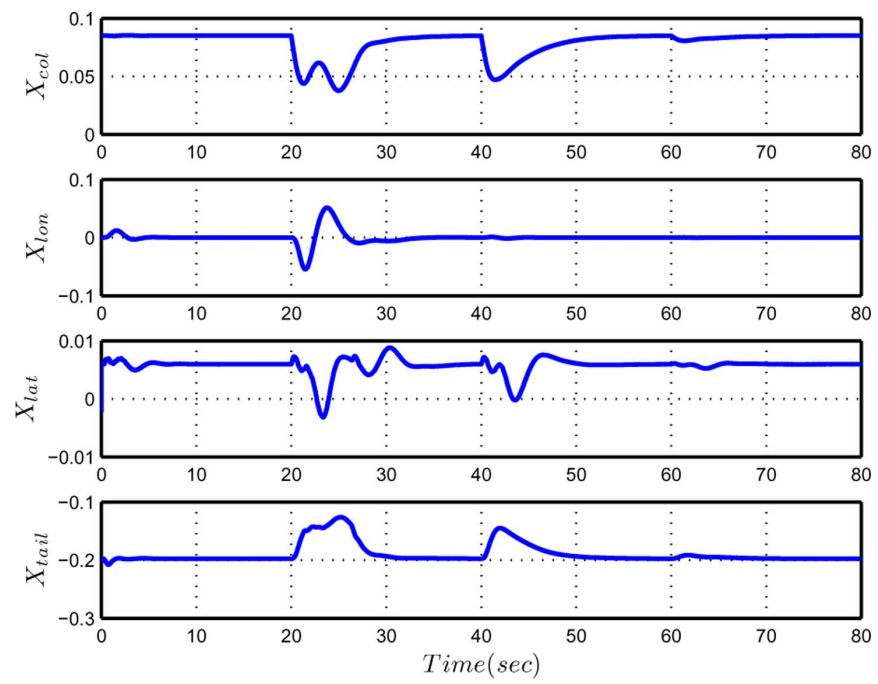

Fig. 6. Control input histories.

in Table II and 3, root mean squared errors of $e_{x}, e_{y}, e_{z}, e_{p s i}$ and root mean squared control efforts have degraded due to the omission of the NN feedforward terms and the increment of the RISE feedback gains. In those simulations, velocity, angular rate, Euler angle and position states are corrupted with sensor measurement noise (having zero mean and $1.5 \mathrm{~m} / \mathrm{s}, 3 \mathrm{deg} / \mathrm{s}, 3$ $\mathrm{deg}, 1.5 \mathrm{~m}$ variances, respectively) and 4 sampling time delays (100 Hz sampling rate).

\section{CONCLUSION}

An LDI-based NN adaptive controller is developed with the RISE feedback for autonomous flight of a RUAV. While the altitude controller in the outer-loop control system is designed by
TABLE II

ROOT MEAN SQUARED CONTROL EFFORTS

\begin{tabular}{|c||c|c|c|c|}
\hline & $X_{\text {col }}$ & $X_{\text {lon }}$ & $X_{\text {lat }}$ & $X_{\text {tail }}$ \\
\hline Only RISE feedback & 0.0803 & 0.0111 & 0.0119 & 0.1901 \\
\hline RISE feedback with NN & 0.0803 & 0.0100 & 0.0066 & 0.1900 \\
\hline
\end{tabular}

the LDI-based RISE feedback control, the inner-loop attitude control system requires the $\mathrm{NN}$ feedforward term to reduce the load of the RISE feedback for eliminating the uncertainty of the flapping angle dynamics. By utilizing the RISE feedback, semi-global asymptotic tracking of the NN-based adaptive control for the RUAV has been enabled with continuous control. Moreover, an adaptive rule for permitting zero initial values of the weight matrices of the NN estimator has been designed by adding the NN feedforward term. Finally, numerical simulation for the automatic landing of the RUAV has been performed to validate the effectiveness of the proposed algorithm.

\section{APPENDIX}

By injecting the control laws (22) and (29) to (20) and (26), the closed-loop error system is obtained as

$$
r_{z}=S_{z}-\mu_{z}, \quad \mathbf{r}_{a}=\hat{\Delta}_{a}^{d}-\Delta_{a}^{d}+S_{a}-\mu_{a}
$$

Then, their time derivatives are determined as

$$
\begin{aligned}
\dot{r}_{z}= & \dot{S}_{z}-\dot{\mu}_{z}=\dot{S}_{z}-\left(k_{z}^{S}+1\right) r_{z}-\beta_{z} \operatorname{sgn}\left(v_{z}\right) \\
\dot{\mathbf{r}}_{a}= & \dot{\hat{\Delta}}_{a}^{d}-\dot{\Delta}_{a}^{d}+\dot{S}_{a}-\dot{\mu}_{a} \\
= & \dot{\hat{W}}^{T} \hat{\sigma}+\hat{W}^{T} \hat{\sigma}^{\prime} \dot{\hat{V}}^{T} \mathbf{x}_{d}+\hat{W}^{T} \hat{\sigma}^{\prime} \hat{V}^{T} \dot{\mathbf{x}}_{d} \\
& -W^{T} \sigma^{\prime} V^{T} \dot{\mathbf{x}}_{d}-\dot{\epsilon}+\dot{S}_{a}-\dot{\mu}_{a}
\end{aligned}
$$

where $\dot{\mu}_{a}=\left(K_{a}^{S}+\rrbracket_{3}\right) \mathbf{r}_{a}+\beta_{a_{1}} \operatorname{sgn}\left(\mathbf{e}_{a_{2}}\right)$.

To facilitate the subsequent stability analysis, we add and subtract a new signal $N_{z}^{d}\left(\triangleq k_{z}^{D} \dddot{z}_{\text {ref }}^{S}-k_{z}^{D} \dot{\Delta}_{w}^{d}\left(z_{\text {ref }}^{S}, \dot{z}_{\text {ref }}^{S}, \ddot{z}_{\text {ref }}^{S}\right)\right)$ to (36) as

$$
\begin{aligned}
\dot{r}_{z} & =\dot{S}_{z}-\left(k_{z}^{S}+1\right) r_{z}-\beta_{z} \operatorname{sgn}\left(v_{z}\right)+N_{z}^{d}-N_{z}^{d} \\
& =\tilde{N}_{z}-v_{z}-\left(k_{z}^{S}+1\right) r_{z}-\beta_{z} \operatorname{sgn}\left(v_{z}\right)+N_{z}^{d}
\end{aligned}
$$

where $\Delta_{w}^{d}(\cdot)$ is $\mathcal{C}^{2}$, and $\tilde{N}_{z}$ is represented as

$$
\tilde{N}_{z}=\dot{S}_{z}-N_{z}^{d}=v_{z}+k_{z} \dot{v}_{z}+k_{z}^{P} \ddot{e}_{z}+k_{z}^{D} \dot{\Delta}_{w}^{d}-k_{z}^{D} \dot{\Delta}_{w} .
$$

In (39), $N_{z}^{d}$ and $\dot{N}_{z}^{d}$ are $\mathcal{L}_{\infty}$ since $\Delta_{w}^{d}$ is $\mathcal{C}^{2}$.

In a similar manner, the terms $\hat{W}^{T} \hat{\sigma}^{\prime} V^{T} \dot{\mathbf{x}}_{d}+\tilde{W}^{T}(\hat{\sigma}-$ $\left.\hat{\sigma}^{\prime} \hat{V}^{T} \dot{\mathbf{x}}_{d}\right)$ are added and subtracted to (37). Then, (32) becomes

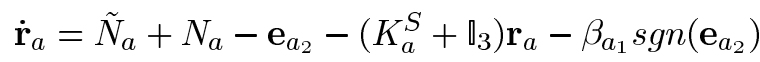


where $\tilde{N}_{a}$ and $N_{a}$ are defined as

$$
\begin{aligned}
\tilde{N}_{a}= & \dot{S}_{a}+\mathbf{e}_{a_{2}}+\operatorname{Proj}\left(\hat{W}, \Gamma_{1}\left(\hat{\sigma}-\hat{\sigma}^{\prime} \hat{V} \dot{\mathbf{x}}_{d}\right) \mathbf{e}_{a_{2}}^{T}\right)^{T} \hat{\sigma} \\
& +\hat{W}^{T} \hat{\sigma}^{\prime} \operatorname{Proj}\left(\hat{V}, \Gamma_{2} \dot{\mathbf{x}}_{d}\left(\hat{\sigma}^{\prime T} \hat{W} \mathbf{e}_{a_{2}}\right)^{T}\right)^{T} \mathbf{x}_{d}, \\
N_{a}= & N_{a}^{d}+N_{a}^{b} \\
N_{a}^{d}= & -\dot{\epsilon}-W^{T} \sigma^{\prime} V^{T} \dot{\mathbf{x}}_{d} \\
N_{a}^{b}= & N_{a}^{b 1}+N_{a}^{b 2} \\
N_{a}^{b 1}= & \hat{W}^{T} \hat{\sigma}^{\prime} V^{T} \dot{\mathbf{x}}_{d}+\tilde{W}^{T}\left(\hat{\sigma}-\hat{\sigma}^{\prime} \hat{V}^{T} \dot{\mathbf{x}}_{d}\right) \\
N_{a}^{b 2}= & -\left(\hat{W}^{T} \hat{\sigma}^{\prime} \tilde{V}^{T} \dot{\mathbf{x}}_{d}+\tilde{W}^{T}\left(\hat{\sigma}-\hat{\sigma}^{\prime} \hat{V}^{T} \dot{\mathbf{x}}_{d}\right)\right) .
\end{aligned}
$$

Then, since $\dot{S}_{z}$ and $\dot{S}_{a}$ are continuously differentiable, we obtain the following inequality by the Mean-Value Theorem [20]:

$$
\begin{aligned}
& \left\|\tilde{N}_{z}(t)\right\| \leq \rho_{z}\left(\left\|\mathbf{z}_{z}\right\|\right)\left\|\mathbf{z}_{z}\right\| \\
& \left\|\tilde{N}_{a}(t)\right\| \leq \rho_{a}\left(\left\|\mathbf{z}_{a}\right\|\right)\left\|\mathbf{z}_{a}\right\|
\end{aligned}
$$

where $\mathbf{z}_{z}=\left[e_{z} v_{z} r_{z}\right]^{T}\left(\in \Re^{3}\right), \mathbf{z}_{a}=\left[\mathbf{e}_{a_{1}}^{T} \mathbf{e}_{a_{2}}^{T} \mathbf{r}_{a}^{T}\right]^{T}\left(\in \Re^{9}\right)$, and $\rho_{z}\left(\left\|\mathbf{z}_{z}\right\|\right), \rho_{a}\left(\left\|\mathbf{z}_{a}\right\|\right)$ are globally invertible, nondecreasing functions.

In a similar manner as in [18], the following inequalities hold:

$$
\begin{aligned}
\left\|N_{z}^{d}(t)\right\|_{\mathcal{L}_{\infty}} & \leq \zeta_{z_{1}} \\
\left\|\dot{N}_{z}^{d}(t)\right\|_{\mathcal{L}_{\infty}} & \leq \zeta_{z_{2}} \\
\left\|N_{a}^{d}(t)\right\|_{\mathcal{L}_{\infty}} & \leq \zeta_{a_{1}} \\
\left\|N_{a}^{b}(t)\right\|_{\mathcal{L}_{\infty}} & \leq \zeta_{a_{2}} \\
\left\|\dot{N}_{a}^{d}(t)\right\|_{\mathcal{L}_{\infty}} & \leq \zeta_{a_{3}} \\
\left\|\dot{N}_{a}^{b}(t)\right\|_{\mathcal{L}_{\infty}} & \leq \zeta_{a_{4}}+\zeta_{a_{5}}\left\|\mathbf{e}_{a_{2}}\right\|
\end{aligned}
$$

where $\|\cdot\|_{\mathcal{L}_{\infty}}$ is the $\mathcal{L}_{\infty}$ norm, and $\zeta_{a_{i}}(i=1,2)$ and $\zeta_{a_{j}}(j=$ $1, \ldots, 5)$ are real positive constants.

Theorem 1: The altitude and attitude control laws, $X_{\mathrm{col}}$ and $\mathbf{u}_{a}$, guarantee that the altitude and attitude errors, $e_{z}$ and $\mathbf{e}_{a_{1}}$, go to zero if the RISE feedback gains $\left(k_{z}^{S}, K_{a}^{S}\right)$ are sufficiently large, and $\beta_{z}$, the diagonal terms of the matrix $\beta_{a_{1}}$ and $\beta_{a_{2}}$ are selected by the following sufficient conditions:

$$
\begin{aligned}
\beta_{z} & >\zeta_{z_{1}}+\frac{1}{k_{z}} \zeta_{z_{2}} \\
\beta_{a_{1 i}} & >\zeta_{a_{1}}+\zeta_{a_{2}}+\frac{1}{k_{2 i}} \zeta_{a_{3}}+\frac{1}{k_{2 i}} \zeta_{a_{4}},(i=1,2,3), \\
\beta_{a_{2}} & >\zeta_{a_{5}}
\end{aligned}
$$

where $\zeta_{a_{i}}(i=1,2)$ and $\zeta_{a_{j}}(j=1, \ldots, 5)$ are given in (46) and (47), $k_{2 i}(i=1, \ldots, 3)$ are the diagonal terms of $K_{2}$ in (24), and $\beta_{a_{2}}$ is introduced in the proof.

Proof: See the details in [18] and [20].

Remark 1: The proposed control system in Fig. 1, which is composed of (22) and (29), ensures asymptotic altitude and attitude tracking using the RISE feedback and NN feedforward terms. $\phi_{\text {ref }}$ and $\theta_{\text {ref }}$ in (16) are generated using the given position $\left(x_{\text {ref }}^{S}, y_{\text {ref }}^{S}, z_{\text {ref }}^{S}\right)$, velocity $\left(\dot{x}_{\text {ref }}^{S}, \dot{y}_{\text {ref }}^{S}, \dot{z}_{\text {ref }}^{S}\right)$ and heading commands $\left(\psi_{\text {ref }}\right)$. Therefore, $e_{x}, e_{y}$ in (17) go to zero.

\section{REFERENCES}

[1] B. L. Stevens and F. L. Lewis, Aircraft Control and Simulation, 2rd ed. New York: Wiley, 2003.

[2] A. J. Calise and R. T. Rysdyk, "Nonlinear adaptive flight control using neural networks," IEEE Control Syst. Mag., vol. 18, no. 6, pp. 14-25, Dec. 1998.

[3] B. Mettler, V. Gavrilets, and E. Feron, "Nonlinear model for a smallsized acrobatic helicopter," presented at the AIAA Guid., Nav., Control Conf., Montreal, QC, Canada, 2001.

[4] B. Mettler, M. B. Tischler, and T. Kanade, "System identification of small-size unmanned helicopter dynamics," presented at the 55th Forum Amer. Hel. Soc., Montreal, QC, Canada, 1999.

[5] B. Mettler, Identification Modeling and Characteristics of Miniature Rotorcraft. Norwell, MA: Kluwer, 2002.

[6] D. H. Shim, T. J. Koo, F. Hoffmann, and S. Sastry, "A comprehensive study of control design for an autonomous helicopter," presented at the Conf. Decision Control, Tampa, FL, Dec. 1998.

[7] D. H. Shim and H. J. Kim, "A flight control system for aerial robots: Algorithms and experiments," IFAC Control Eng. Prac. (CEP), vol. 11, no. 12 , pp. 1389-1400, Oct. 2003.

[8] H. J. Kim, D. H. Shim, and S. Sastry, "Nonlinear model predictive tracking control for rotorcraft-based unmanned aerial vehicles," presented at the Amer. Control Conf., Anchorage, AK, May 2002.

[9] D. H. Shim, H. J. Kim, and S. Sastry, "Decentralized nonlinear model predictive control of multiple flying robots in dynamic environments," presented at the Conf. Decision Control, Maui, HI, 2003.

[10] E. N. Johnson and S. K. Kannan, "Adaptive flight control for an autonomous unmanned helicopter," presented at the AIAA Guid., Nav., Control Conf., Monterey, CA, 2002.

[11] E. N. Johnson and S. K. Kannan, "Adaptive trajectory control for autonomous helicopters," J. Guid., Control Dyn., vol. 28, no. 3, pp. 524-538, 2005.

[12] K. P. Tee, S. S. Ge, and F. E. H. Tay, "Adaptive neural network control for helicopters in vertical flight," IEEE Trans. Control Syst. Technol., vol. 16, no. 4, pp. 753-762, Mar. 2008.

[13] A. Y. Ng, H. J. Kim, M. I. Jordan, and S. Sastry, "Autonomous helicopter flight via reinforcement learning," presented at the NIPS 16, Vancouver and Whistler, BC, Canada, 2004.

[14] P. A. Ioannou, Robust and Adaptive Control. Upper Saddle River, NJ: Prentice-Hall PTR, 1996.

[15] H. K. Khalil, Nonlinear Systems, 3rd ed. Upper Saddle River, NJ: Prentice-Hall PTR, 2002

[16] J. Shin, H. J. Kim, Y. Kim, and W. E. Dixon, "Attitude tracking of the rotorcraft-based UAV via RISE feedback and NN feedforward," presented at the Conf. Decision Control, Atlanta, GA, 2010.

[17] P. M. Patre, W. MacKunis, C. Makkar, and W. E. Dixon, "Asymptotic tracking for systems with structured and unstructured uncertainties," IEEE Trans. Control Syst. Technol., vol. 16, no. 2, pp. 373-379, Mar. 2008.

[18] P. M. Patre, W. MacKunis, K. Kaiser, and W. E. Dixon, "Asymptotic tracking for uncertain dynamic systems via a multilayer neural network feedforward and rise feedback control structure," IEEE Trans. Autom. Control, vol. 53, no. 9, pp. 2180-2185, Oct. 2008.

[19] W. MacKunis, P. M. Patre, M. K. Kaiser, and W. E. Dixon, "Asymptotic tracking for aircraft via robust and adaptive dynamic inversion methods," IEEE Trans. Control Syst. Technol., vol. 18, no. 6, pp. 1448-1456, Nov. 2010.

[20] B. Xian, D. M. Dawson, M. S. de Qeuiroz, and J. Chen, "A continuous asymptotic tracking control strategy for uncertain nonlinear systems," IEEE Trans. Autom. Control, vol. 49, no. 7, pp. 1206-1211, Jul. 2004.

[21] C. Makkar, G. Hu, W. G. Sawyer, and W. E. Dixon, "Lyapunov-based tracking control in the presence of uncertain nonlinear parameterizable friction," IEEE Trans. Autom. Control, vol. 52, no. 10, pp. 1988-1994, Oct. 2007.

[22] Z. Cai, M. S. de Queiroz, and D. M. Dawson, "Robust adaptive asymptotic tracking of nonlinear systems with additive disturbance," IEEE Trans. Autom. Control, vol. 51, no. 3, pp. 524-529, Mar. 2006.

[23] J. V. R. Prasad and A. M. Lipp, "Synthesis of a helicopter nonlinear flight controller using approximate model inversion," Math. Com. Model., vol. 18, no. 3, pp. 89-100, Aug. 1993.

[24] Y. I. Kim, S. C. Lee, and B. Kim, "A study on helicopter trajectory tracking control using neural networks," Korean Soc. Aero. Space Sci. (In Korean), vol. 31, no. 4, pp. 50-57, Apr. 2003.

[25] B. J. Park, C. H. Hong, and J. Y. Suk, "Adaptive output feedback control of unmanned helicopter using neural networks," (in Korean) Korean Soc. Aero. Space Sci., vol. 35, no. 11, pp. 990-998, Nov. 2007. 